\title{
Wissenschaft schafft Fakten und interpretiert sie - Weisheit kommt von anderswo
}

Jürg Kesselring

Korrespondenz:

Prof. Dr. med. Jürg Kesselring Chefarzt Neurologie

Rehabilitationszentrum

CH-7317 Valens

Tel. 0813031408

Fax 0813031410

kesselring.klival[at]spin.ch
Hermann von Helmholtz (1821-1894), einer der einflussreichsten Wissenschaftler und Philosophen im 19. Jahrhundert, prägte die spannenden Zeiten des Übergangs von Aufklärung und Romantik bis in die Anfänge der modernen Neurowissenschaften mit und setzte mit seinem Werk neue Perspektiven. In seinem Nachruf heisst es: «Zu seinem unbestrittenen beruflichen Erfolg trug Helmholtz eine lange Reihe von Artikeln und Büchern bei. Es wurde gesagt, so wie sieben Städte sich um Homer stritten, so beanspruchen die sieben Wissenschaften, Mathematik, Physik, Chemie, Physiologie, Medizin, Philosophie und Ästhetik, Helmholtz als einen der ihrigen und es ist interessant zu beobachten, wie früh er eine umfassende Sicht der Wissenschaft wahrnahm, die diese rhetorische Aussage rechtfertigt. Vielfalt und Bedeutung seines Werkes sicherten ihm zu Lebzeiten die Bewunderung und den Respekt der ganzen zivilisierten Welt [...] alle schätzen die Leistungen, die er in der Biologie dank der Anwendung seines mathematischen Genies und gut ausgebildeter Fähigkeiten zu experimenteller Forschung in physiologischen Untersuchungen gemacht hat. Seine frühen natürlichen Neigungen galten der Physik, aber sein Vater konnte ihm die Möglichkeit eines Physikstudiums nicht bieten, sodass er Medizin studierte, um seinen Lebensunterhalt zu verdienen, und er selbst war keineswegs abgeneigt, die lebende statt der toten Materie zu studieren. Wir können jetzt besser verstehen, wie viel die Welt dadurch gewonnen hat, dass dieser junge Mann von grossem Genie und Kraft, einen praktischen Beruf wählen musste. Ein frühes Ergebnis war 1847 sein grosser Essay «Über die Erhaltung der Kraft», mitgeteilt vor der Physikalischen Gesellschaft zu Berlin am 23. Juli 1847, worüber er im Jahr 1891 sagte: `Mein Ziel war es lediglich, einige kritische Untersuchungen und Zusammenstellung von Fakten zugunsten der Physiologen zu geben.> Sein Essay liefert gute Argumente für die Ablehnung der Theorie der ¿Lebenskraft, wurde zwar von einigen der physikalischen und philosophischen Autoritäten jener Zeit als eine phantastische Spekulation abgetan, aber von jüngeren Schüler-Philosophen begeistert begrüsst und bald mussten die älteren Männer überzeugt werden, dass die tatsächliche Wirksamkeit der Vitalität, so gross und schön sie ist, nicht ohne eine Quelle der Energie auskommt» [1].

In seinem Essay «Goethe und die Wissenschaften» bestimmt der deutsche Dichter und Arzt Gottfried Benn (1886-1956) in poetischer Genauigkeit exakt den Beginn der mathematischen und physikalischen Wissenschaft, die sich nach einigen Reinigungsprozessen radikal von der Naturphilosophie und der vitalistischen Physiologie entfernt: «Die eigentliche Geburt dieser neuen Weltsicht war der 23. Juli 1847, an der Tagung der Physikalischen Gesellschaft zu Berlin, in der Helmholtz, angeregt durch Robert Mayer, Berechnungen und Überlegungen zum Problem der Erhaltung der mechanischen Energie als ein allgemeines Gesetz der Natur zeigte. An diesem Tag begann die Idee der vollständigen Verstehbarkeit

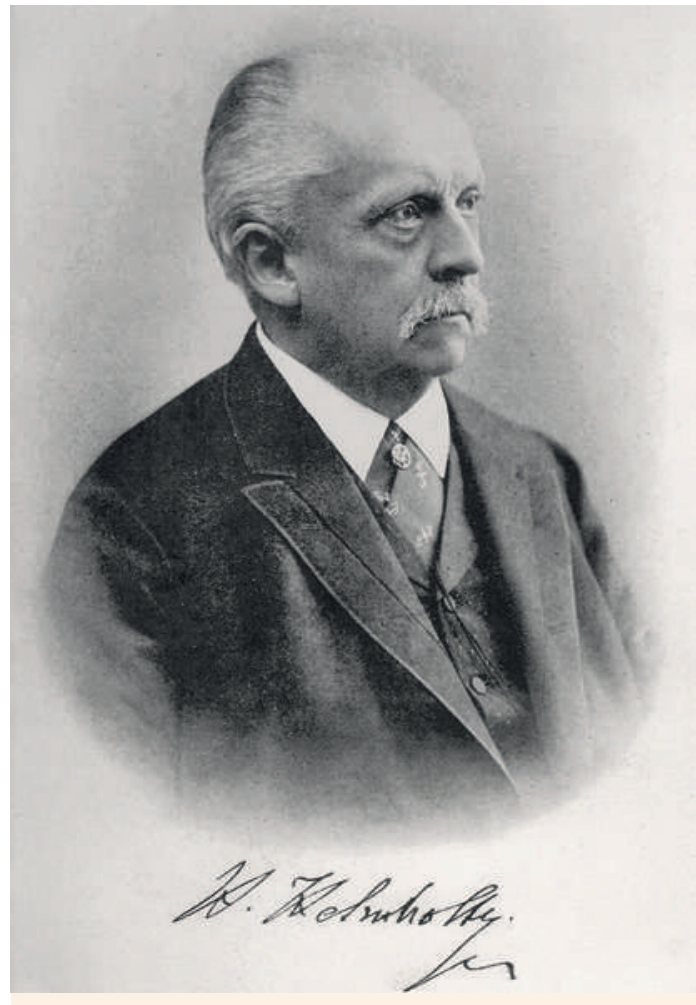

Hermann von Helmholtz lebte von 1821 bis 1894 und war einer der einflussreichsten Wissenschafter und Philosophen im 19. Jahrhundert. 
der Welt als Mechanismus» [2]. Helmholtz hat sich zwar mehrmals deutlich und unmissverständlich vom philosophischen Materialismus distanziert, dennoch aber kann gezeigt werden, «dass er 1 . in seiner Wissenschaftstheorie von einer metaphysischen Position ausgegangen ist, die er nie ganz abstreifen konnte und 2., dass sowohl Helmholtz' Reduktionismus als auch seine Erkenntnistheorie Wurzeln in der Philosophie des Deutschen Idealismus besitzen. Obwohl sein philosophisches Denken einen tiefgreifenden Wandel erfuhr, blieb es in einen idealistischen und metaphysischen Rahmen eingebettet» [3]. In seiner Schrift «Über die Erhaltung der Kraft», die dem Vortrag folgte, geht Helmholtz davon aus, dass die Erscheinungen in der Natur das Resultat verborgener Ursachen darstellen. Diese sind mit dem Vermögen ausgestattet, Wirkungen aufeinander und auf unsere Sinnesorgane auszuüben. Dieses Vermögen nennt Helmholtz «Kraft», bestimmt sie als «unveränderliche Bewegungskraft. Das Ziel der Naturwissenschaft, die Natur zu begreifen, besteht darin, die Erscheinungen zurückzuführen auf unveränderliche, anziehende und abstossende Kräfte, deren Intensität von der Entfernung abhängt.» Er wollte «das Reale in zwei Abstractionen, Materie und Kraft, logisch auflösen [...] Das Vergängliche ist aufzufassen als die Erscheinungsform des Unvergänglichen, des Gesetzes» [3].

Helmholtz selbst sah die Gefahr solcher einseitiger Entwicklungen in seiner späteren Rede «Das Denken in der Medizin» (1877): «Sind wir mit den Auffassungen von l'homme machine der materialistischen Aufklärung als der Scylla der Naturphilosophie entgangen, so besteht jetzt die Bedrohung der Charybdis des Fallens in den reinen Materialismus: Unsere Generation hat noch unter dem Druck der spiritualistischen Metaphysik gelitten, die jüngere Generation läuft in die Gefahr des reinen Materialismus» [4]. Helmholtz erlebte und prägte eine Zeit, die einen

\section{Für den Dichter Goethe bedeutete die Verwendung von Laborgeräten ein «Quälen der Natur, während man sie mit Respekt und Rücksicht behandeln sollte wie ein Kunstwerk».}

Wendepunkt in der Geschichte darstellt. Sie ist sozusagen als ein Zeitraum von höchster Geschwindigkeit einer Schaukel zwischen zwei verschiedenen Modi, die Welt zu betrachten und in ihr zu handeln. Dies hat vor kurzem Iain McGilchrist in seinem monumentalen Buch «The Master and his Emissary» ausführlich dargestellt: «The Divided Brain and the Making of the Western World» $[5,6]$.

Helmholtz schrieb zwei Aufsätze über die wissenschaftlichen Arbeiten von Johann Wolfgang von Goethe: «Über Goethes naturwissenschaftliche Arbei- ten» (1853) und «Goethes Vorahnungen kommender naturwissenschaftlicher Ideen» $(1892)$ [7, 8]. In beiden Artikeln geht es um die Frage, was richtige Wissenschaft sei im Gegensatz zur Kunst im Kontext von Goethes Werk in der Morphologie, Botanik und Optik. Goethe als Prosaschreiber, vor allem aber als Dichter, mit universeller Neugier und breiter, oft vi-

\section{«Das Vergängliche ist aufzufassen als die Erscheinungsform des Unvergänglichen, des Gesetzes.»}

sionärer Kultur, immer sicher seines eigenen Wertes, war überzeugt, dass die Natur Lektionen des Lebens erteilte und dass die Wissenschaft interpretieren soll, statt sich nur in den Dienst der Technik zu stellen. Er sang ein Loblied auf die «tiefe Harmonie» der Natur. Helmholtz' Ansichten über Wissenschaft und seine Sorgen über Goethe enthüllen Details der vermeintlichen Dichotomie zwischen Kunst und Wissenschaft. Die methodischen Grenzen der Disziplinen folgen nicht einfach aus der Definition ihrer Projekte, sondern ihre Projekte und Methoden werden durch Diskurse und Praktiken in spezifischen politischen und kulturellen Kontexten artikuliert, die sich in Bezug auf Vorstellungen von anderen Praktiken oft unterscheiden [9]. In der früheren Arbeit zu Goethes wissenschaftlichen Untersuchungen schrieb Helmholtz: «Anstatt zu versuchen, die Phänomene der Natur unter bestimmten Definitionen, unabhängig von Intuition, zu untersuchen, setzt er sich ihnen gegenüber, als würde er ein in sich abgeschlossenes Kunstwerk betrachten, das seine zentrale Idee, früher oder später einem ausreichend empfänglichen Studenten offenbaren würde» [7]. Helmholtz argumentiert, dass Goethes Werk nicht richtig wissenschaftlich sei, weil er die Natur mit den intuitiven Augen des Künstlers sah, was zu zwei unentschuldbaren Fehlern führe: 1. das Versäumnis, kausale Zusammenhänge zu erfassen, und 2. zu vernachlässigen, klare und eindeutige Theorien zu formulieren. Wissenschaft sei auf Gesetze gegründet, generalisierte Formeln, die genau die invarianten kausalen Beziehungen zwischen Erscheinungen angeben. Er befürwortete eine «experimentelle interaktionistische» Konzeption von Wissenschaft, beginnend mit Verallgemeinerungen über Erfahrung und Manipulation von Variablen, um zu sehen, was unter unterschiedlichen Versuchsbedingungen konstant blieb, dann die Verfeinerung der ursprünglichen Hypothese, bis zu einem genauen Ausdruck der Beziehungen von gut definierten Gruppen von Objekten [9].

In seinem späteren Aufsatz «Goethes Vorahnungen kommender naturwissenschaftlicher Ideen» vertrat Helmholtz weiter die Auffassung, dass künstle- 
rische Intuition ein passiver Vorgang sei, durch den der Künstler allgemeine Wahrheiten über makrokosmische Erfahrungen gewinnen konnte, und gab zu, dass Intuitionen oft der erste Schritt in einem wissenschaftlichen Projekt seien. Er behauptet sogar, dass die Kunst «eine andere Art und Weise sei, ausserhalb der Wissenschaft, Einblick in die komplizierte Funktionsweise der Natur zu erwerben" [8]. Er argumentierte, dass in dieser Hinsicht Goethe gewisse zeitgenössische wissenschaftliche Theorien vorweggenommen habe und zitiert das Werk des Dichters als weniger präzise Vorwegnahme seiner eigenen Theorie der Empfindung und vom Gesetz von der Erhaltung der Kraft. Für Helmholtz war Goethe als Wissenschaftler gescheitert, nicht weil sein übermässig aktiver, romantischer Wille sich auf seine Darstellungen der Natur projizierte, sondern vielmehr weil Goethe in seinem passiv beobachtenden Ansatz die Natur einfach als gegeben annahm - das war für Helmholtz der Ursprung von Goethes wissenschaftlichen Schwächen. Der Künstler nahm an, dass die Wahrheiten der Natur einfach durch einen hinreichend subtilen Geist erfasst werden können.

Helmholtz empfand eine Mission, die Naturphilosophie mit ihrem «Verweis auf Autorität und unbegrenzte Spekulation» von ihrer privilegierten Position in der deutschen Wissenschaft zu entfernen und die experimentelle Wissenschaft an ihren Platz zu stellen. Gegen die Übel der Naturphilosophie verordnete er die «Praktiken zur Kultivierung unermüdlicher Geduld, die strikte Einhaltung der experimentellen Methoden und Schritt-für-Schritt-Argumentation,

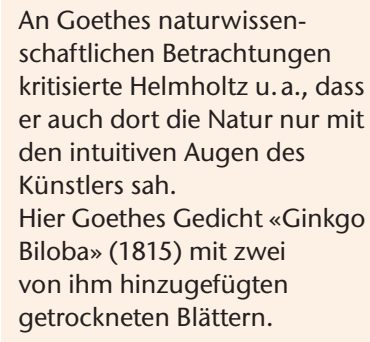

\section{Ginkgo Biloba}

Dieses Baums Blatt, der von Osten

Meinem Garten anvertraut, Giebt geheimen Sinn zu kosten,

Wie's den Wissenden erbaut, Ist es Ein lebendig Wesen,

Das sich in sich selbst getrennt?

Sind es zwey, die sich erlesen, Dass man sie als Eines kennt?

Solche Frage zu erwiedern,

Fand ich wohl den rechten

Sinn,

Fühlst du nicht an meinen Liedern,

Dass ich Eins und doppelt bin? um zu verhindern, dass vorschnell auf Schlussfolgerungen gesprungen würde». Nur durch sorgfältige und geduldige Durchführung von Tests und durch die sorgfältige Anhäufung von Beweisen könne ein tüchtiger Wissenschaftler erreichen, eine blosse Hypothese in den Status eines Gesetzes zu erheben.

Warum war Helmholtz, einer der angesehensten Wissenschaftler seiner Zeit in Deutschland, an den wissenschaftlichen Untersuchungen Goethes so sehr interessiert, der sich selbst in erster Linie als Dichter sah? «Der Wissenschaftler in ihm verhinderte die Zerstörung des Menschen in ihm: Für sein Bedürfnis nach Schönheit und Kultur und seinen offensichtlichen Wunsch, seine Gefühle zu teilen [...] und für die Ausarbeitung der wissenschaftlichen Argumentation im Gegensatz zur Metaphysik fand er die Philosophie äusserst wichtig. Er machte den Unterschied zwischen der logischen Argumentation des Wissenschaftlers und dem poetischen Denken des Dichters, von denen jedes eine eigene Methodik und unterschiedliche Ziele hatte.» [10]. Helmholtz erkannte, dass «der Dichter ganz konkret zum Erwerb von Wissen beitragen kann [...] dank der Kraft seiner künstlerischen Intuition und seiner metaphorischen Visionen» [10]. Er kann Zustände des Geistes mit einer Wahrhaftigkeit beschreiben, die für Philosophen oder empirische Wissenschaftler unzugänglich sind. Helmholtz hat auch, wie so viele seiner Zeitgenossen (sicherlich in Deutschland) diesen inneren Zwiespalt gefühlt und erlebt, wie er oft aus Goethes Faust I, Verse 1112 bis 1117 zitiert wird:

«Zwei Seelen wohnen, ach! in meiner Brust, die Eine will sich von der andern trennen; die Eine hält in Derber Liebeslust sich an die Welt mit klammernden Organen; die and're hebt gewaltsam sich vom Dust zu den Gefilden Hoher Ahnen»

Helmholtz erkannte, dass Künstler auf einer anderen Ebene des Bewusstseins als Wissenschaftler arbeiten und dass die einer künstlerischen Intuition offenbarten Wahrheiten über den menschlichen Verstand der wissenschaftlichen Forschung nicht in gleicher Weise zugänglich waren. Er betonte, dass sowohl Wissenschaftler als auch Künstler mit ihren je eigenen Methoden in der Lage seien, neue Erkenntnisse zu erwerben. Für den Dichter Goethe bedeutete die Verwendung von Laborgeräten ein «Quälen der Natur, während man sie mit Respekt und Rücksicht behandeln sollte wie ein Kunstwerk» [10].

Helmholtz wusste von seinen eigenen Erfahrungen als Patient während seiner jüngeren Jahre und als praktizierender Arzt, dass «... am Patientenbett die Vernunft allein häufig nicht genügt, das komplizierte Gewirr der vielfachen und verschiedenartigen Ursachen einer Krankheit zu entwirren ..., dass das Gesetz der Kausalität, welches Kant und die Physiker so sehr liebten, sich nicht so einfach auf das Leben anwenden liess» [10]. 
Nach seinen physiologischen Arbeiten über Untersuchungen zur Geschwindigkeit der Übertragung von Nervenimpulsen entlang der Sinnesnerven und der motorischen Nerven zu den Muskeln führte Helmholtz einige Jahre später «seine grosse Arbeit über 〈Tonempfindungen` [11, 12] aus, in der es nicht nur um die Wahrnehmung des Tones, sondern ebenso sehr um mathematische und experimentelle Untersuchungen geht, von der anatomischen Struktur des Ohrs bis zum philosophischen Fundament der Musikkunst - ein Werk, das eine einzigartige Position in der Literatur der Philosophie hält, und sicher ein herrliches Denkmal für das Genie und die unbezähmbaren Arbeitsmacht seines Autors ist» [1]. Helmholtz machte die Ästhetik der Tonkunst nach einem Jahrhundert der Vernachlässigung zum Gegenstand seiner Untersuchungen, als er schrieb: «Musik hat bisher sich von der wissenschaftlichen Behandlung mehr zurückgezogen als jede andere Kunst. Dichtung, Malerei und Skulptur borgen von der Welt der Erfahrung, aber die Musik scheint die Zergliederung der Empfindungen zu verunmöglichen.» Als Empiriker verstand er das Vereinigende von Musiktheorie und Ästhetik. Musik könne nur als grosse Kunst verstanden werden, welche Anatomie, Physiologie, Philosophie und Psychologie verbindet [8]. Helmholtz ist ein ausgezeichneter Führer zu geistigen Spaziergängen in diese doppeldeutigen, zum Teil auch zwielichtigen Dämmerzonen der Geschichte, wo Vernunft und künstlerische geistige Anlagen in einzelnen Personen in solch aussergewöhnlichem Masse zusammenkommen [13]. «Zweifellos war er eine der feinsten Federn in der Kappe der neurologischen Wissenschaft im Neunzehnten Jahrhundert, ein unübertroffener Techniker, theoretischer Physiker und ausgezeichneter Mathematiker [...] fähiger Anatom und Physiologe [...] ein erfahrener und begeisterter Kenner der Geschichte der Musik und Musikwissenschaft» [10].

Der fundamentale Mentalitätswandel bahnte sich schon früher an, wie sich in einer Episode um den «Philosophen von Sanssouci» und die Bach-Familie illustrieren lässt: Friedrich der Grosse bat Johann Sebastian Bach, als dieser ihn und seinen Sohn Carl Philipp Emanuel im Jahre 1747 in Potsdam besuchte, über ein Musikthema, welches er selbst komponiert hatte, in seiner Gegenwart Fugen zu improvisieren. Dies tat Johann Sebastian Bach auch tatsächlich sogleich mit Erfolg. Als aber der König von ihm forderte, sechsfache Fugen von diesem Thema zu setzen, war er etwas verlegen, weil er fand, dass das Thema für eine solch schwierige Aufgabe nicht gut genug war. Diese Episode kennzeichnet eine grundlegende Wende in der Geschichte: dass ein Untertan trotz all seiner Verbeugungen und Ehrerbietungen finden und danach handeln konnte, dass sein eigener künstlerischer Wille wichtiger war als der Befehl eines Souveräns. Bach ging nach Hause und setzte dreizehn herrliche Variationen auf ein eigenes Thema und widmete dann die Arbeit dem König, als ob sie auf seinem könig- lichen Thema entstanden sei unter dem Titel, der dem Wort «Ricerca» (Research, Forschung) so nahekommt: «Ricercar», Abkürzung für Regis Iussu Cantio Et Reliqua Canonica Arte Resoluta (das durch Befehl des Königs gegebene Thema mit Hinzufügungen versehen, aufgelöst im kanonischen Stil). Dies ist jetzt als «Das Musikalische Opfer» bekannt [13, 14], wobei «Opfer» in unserer Sprache verschiedene Bedeutungen haben kann, die Bach ohne Zweifel in diesen Titel «hineingeheimnist» hat ...

\section{Literatur}

1 Obituary Hermann von Helmholtz. Proceedings of the Royal Society of London 1896; 59: xvii-Xxx.

2 Jürg Kesselring. Die Geburtsstunde der modernen Neurowissenschaften: Hermann Helmholtz' Vortrag «Über die Erhaltung der Kraft» am 23. Juli 1847 vor der physikalischen Gesellschaft zu Berlin. Schriftenreihe der Deutschen Gesellschaft für Geschichte der Nervenheilkunde. Band 18, 012, S.281-90.

3 Heidelberger M. Helmholtz' Erkenntnis- und Wissenschaftstheorie im Kontext der Philosophie und Naturwissenschaft des 19. Jahrhunderts. In: Krüger L (Hrsg). Universalgenie Helmholtz. Rückblick nach 100 Jahren. Berlin: Akademie-Verlag; 1994. S. 168-85.

4 Helmholtz H. Goethes Vorahnungen kommender naturwissenschaftlicher Ideen. Rede in der Generalversammlung der Goethe Gesellschaft zu Weimar am 11. Juni 1892. In: Mandelkow KR (Hrsg.). Goethe im Urteil seiner Kritiker. Dokumente zur Wirkungsgeschichte Goethes in Deutschland. Teil III: 1870-1918. München: Beck; 1979. S. 227-44.

5 McGilchrist I. The Master and His Emissary. The divided brain and the making of the Western world. New Haven, London: Yale University Press; 2009.

6 Kesselring J. Links und/oder rechts? Schweiz Ärztezeitung. 2012;93(14/15):564-5.

7 Helmholtz H. Über Goethes naturwissenschaftliche Arbeiten. Vortrag zu Königsberg. 1853. Braunschweig. 1896.

8 Rousseau G. A polymath rediscovered. Nature. 2010;467:1039-40.

9 Hallet D. On the Subject of Goethe: Hermann von Helmholtz on Goethe and Scientific Objectivity. Spontaneous Generations: A Journal for the History and Philosophy of Science. 2009;3(1):178-94.

10 Meulders M. Helmholtz. From Enlightenment to Neuroscience. Cambridge: MIT Press; 2010.

11 Helmholtz H. Die Lehre von den Tonempfindungen als physiologische Grundlage für die Theorie der Musik. Reprint von 1863. Frankfurt / M: MinervaVerlag; 1981.

12 Rieger M. Helmholtz Musicus. Die Objektivierung der Musik im 19. Jahrhundert durch Helmholtz' Lehre von den Tonempfindungen. Darmstadt: Wissenschaftliche Buchgesellschaft; 2006.

13 Kesselring J. Twilight at the birth of modern neuroscience. Book Review: Michel Meulders: From Enlightenment to Neuroscience. Brain. 2011;134:1571-4.

14 Schneewind U. «Jede Note an Dich gerichtet!» Musikalische Widmungsgeschichten aus drei Jahrhunderten. Darmstadt: Wissenschaftliche Buchgesellschaft; 2009. 